after infection, this is, 9, 8, 7 and 6 weeks after dosing against the 1-, 2-, 3- and 4-week-old infections, respectively. Superficial scarring of the liver indicated the inicial tracks made by the young flukes invading the liver.

The results of the rabbit experiments are shown in Table 1. Control, untreated and infected rabbits yielded an average 'take' of 7 adult flukes, the range being 1-13 adult flukes.

4,4'-Diaminodiphenylmethane was active against the immature forms at $0.2 \mathrm{~g} / \mathrm{kg}$ when administered intraporitoneally, but inactive by oral administration.

Two infected eattle were given. $0.1 \mathrm{~g} / \mathrm{kg}$ body-weight by drenching bottle 8 months after infection and at post-mortem examination two weeks

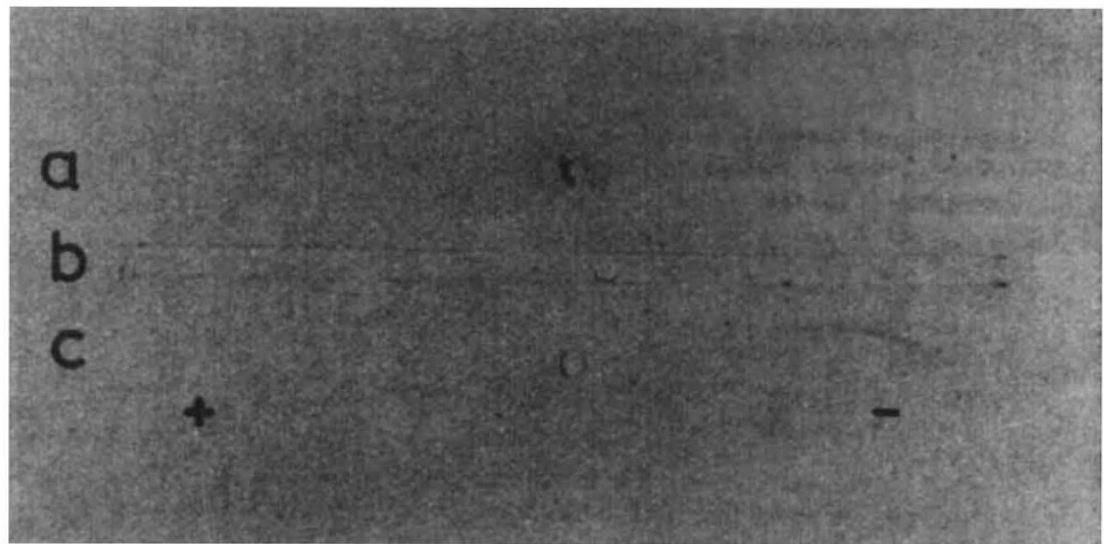

Fig. 1. $a$, Normal control serum; $b$, trough containing $F$. hepatica antigen; $c$, serum from patient infected with $F$, hepatica

later were found to be cleared of flukes; another was given $0.025 \mathrm{~g} / \mathrm{kg}$, which showed no activity, flukes being ob. served at post-mortern examination two weeks after dosing; and one other, given $0 \cdot 1 \mathrm{~g} / \mathrm{kg}$ by subcutaneous injection, was cured. Signs of toxicity observed in these cattle within 14 days after dosing consisted of yellowing and peeling of the nose, ataxia and apparent impairment of vision.

In toxicity tests on twelve uninfected cattle, doses ranging from $0.1 \mathrm{~g} / \mathrm{kg}$ and $1.0 \mathrm{~g} / \mathrm{kg}$ were given by drenching bottle. Doses of $0.2 \mathrm{~g} / \mathrm{kg}$ and greater were fatal, and of the three calves dosed with $0.1 \mathrm{~g} / \mathrm{kg}$, one died after threo weeks and the two survivors showed yellowing of tho sclerotis, sloughing of the skin of the nose and the back, and transient impairment of vision after 3 weeks. All the surviving cattle developed yellowing of the sclerotis, which was not always manifest until about 3-4 weeks after dosing, particularly after the lower dose-levels.

$I$ have confirmed the observation of Lämmler and Loewe ${ }^{4}$ that certain diaminodiphenyl alkanes are effective against the mature $F$. hepatica. I have found that $4,4^{\prime}$-diaminodiphenyl methane hydrochloride is offective against the very early immature forms (1-4. week-old) in rabbits - an observation hitherto unrecorded. Unfortunately the compound showed toxic effects in cattle which would prevent its development as a therapeutic agent.

Veterinary Research Division,

VALERIE N. Williams

May and Baker, Ltd.,

Fyfield Road,

Ongar, Essox.

1 Hughes, D. L., thesis, Univ. London (1959).

2 Parfitt, J., and Kendall, S. B., Brit. Vet. J.,118, 1 (1962).

` Lämmler, G., and Loewe, H., Arzn.-Forsch., 5, 497 (1955).

${ }^{4}$ Lämmler, G., and Loewe, H., Arzn.-Forsch., 12, 15, 164 (1962).

\section{Diagnosis of Liver Fluke (Fasciola hepatica) Infection in Human Beings by Means of Immunoelectrophoresis}

IN the southern parts of Yugoslavia, infection of human beings with parasites such as Echinococcus, Ascaris, Taenia, Trichina, Fasciola and Amoeba is common. The diagnosis of these conditions is difficult, for the clinical picture is not always characteristic. Moreover, positive identification is essential for medication (Taenia) or surgical intervontion (Echinococcus).

These parasites provoke antibody formation in the host, and this has been made the basis of serological and allergological methods for diagnosis, including tho double diffusion method of Ouchterlony ${ }^{1}$. We suggest, however, that immunoelectrophoresis ${ }^{2}$ will give quicker and more reliable results.
The liver fluke ( $F$. hepatica) is found principally in sheep, but it occessionally infects human beings, lodging in the biliary tract and causing damage to the liver. In a child (B. R., aged 9) a diagnosis of fascioliasis was made on a basis of a pronounced sideropenia, eosinophilia (60 per cent), albumin/globulin ratio less than 1 and a positive serological test. The diagnosis was confirmed by the finding of Fasciola eggs in the fæces.

Blood serum from this patient was subjected to electrophoresis in agar-gel and was then allowed to react with a sample of $F$ ' hepatica antigen (Institut 'Merieux', Lyon). A characteristic precipitation arch was produced in the slow $\gamma$-globulin region (Fig. 1). A normal control serum treated simultaneously in the same manner gave no precipitate.

Though we have made many attempts, we have been unable to diagnose echinococcosis by a similar procedure, possibly owing to an unfavourable antigen-antibody relation in our experiments. Attempts to diagnose infection with other parasites by similar means are now in progress.

Clinical Laboratory,

Children's Hospital, Titograd.

\section{TEODOROVIC}

\section{BERKE:}

Institute of Biochemistry,

Faculty of Pharmacy, Beograd.

Institute of Parasitology, Faculty of Medicine, Beograd.

1 Ouchterlony, O., Ann. Allergy, 5, 1 (1958). Kagan, I. G., and Normau, L. Amer. J. Trop. Med. Hyg., 10, 727 (1061).

'Grabar, P., and Williams, jun., C. A., Biochim. Biophys. Acta, 17, 67 (1955).

\section{Effect of Magnetic Fields on Tissue Respiration}

Ir has been known for some time that the behaviour of certain types of biological materials may be influenced by magnetic fields. Lenzi ${ }^{1}$ reported a difference in effect on neoplastic tissue of a constant and an alternating magnetic field. Other workers ${ }^{2-4}$ have shown by various methods the reaction of other tissues to magnetic fields. In an attempt to extend earlier investigations in our labora. tories ${ }^{5}$ and to develop a quantitative method we have conducted a series of experiments on the effects of magnetic fields on tissue respiration.

An Alnico permanent magnet with an average field strength of 7,300 gauss was used in all experiments. The respirometers used were a constant-pressure type ${ }^{6}$ designed for use with very small (1-4 mg) amounts of tissue. 